\title{
Simulation of Frequency Hopping Communication System Based On MATLAB
}

\author{
Xiaoping $\mathrm{Xu}^{1}$ Weiqi Wang ${ }^{2}$ Lan $\mathrm{Yi}^{3}$ Jie Rong $^{4}$ Anqi Wang ${ }^{5}$ \\ ${ }^{1,2.3 .4 .5}$ Electronic Information and Control Engineering,Beijng university of technology, China
}

\begin{abstract}
In today's information age, how to carry out the accurate information communication is a crucial issue in the field of communication, which is widely used in civil and military fields. Frequency hopping communication system is a typical spectrum communication system, it is in military communication, mobile communication, computer wireless data transmission and wireless LAN and other fields have a very wide range of applications, has become the current short wave communication is one of the important development directions. In this paper, we introduce a design of frequency hopping communication system based on Matlab.Through the frequency hopping communication system the simulation research and theoretical analysis, to achieve the desired results.
\end{abstract}

\section{Background}

Spread spectrum communication has very strong anti-interference ability and security, first appeared in the military field. In recent years, spread spectrum communication is also widely used in the field of civil. Spread spectrum communication has two ways, they are the direct sequence spread spectrum (DSSS) and frequency hopping spread spectrum (FHSS).

\section{The Working Principle and Characteristics of Spread Spectrum Communication}

In the information theory, the channel capacity formula of the channel capacity formula is $\mathrm{C}=\mathrm{w} \log _{2}(1+\mathrm{SNR})$

This is the Shannon's capacity formula. C is the channel capacity (limit speed data transmission)W is the signal bandwidth and SNR is the signal to noise ratio.

The formula shows that the signal to noise ratio and the signal bandwidth can be the same as the transmission rate can be changed. We can improve the signal bandwidth to reduce the signal to noise ratio requirements, Thus enhancing the ability to resist interference. By expanding the signal bandwidth, the ability to resist interference is also improved.

As mentioned above, spread spectrum communication through the expansion of signal bandwidth, in exchange for a strong anti-interference ability, which is the biggest characteristic of the spread spectrum communication. In addition to the signal spectrum expansion, its power in the entire extended frequency potential, but also become sparse and close to the uniform distribution, which increases the difficulty of the third party capture. Who do not know the synchronization of spread spectrum signal of third-party recipients, spread spectrum signal as buried in the noise as it is difficult to detect. In addition, as well as to improve the time resolution of spread spectrum communication and multi access etc.. So spread spectrum communication technology has become a hot spot of research.

One of the important parameters of spread spectrum communication is spread spectrum gain, which reflects the system's ability to resist interference. It is a measure to improve the SNR.

$$
\mathrm{G}=\mathrm{SNR}_{\mathrm{wat}} / \mathrm{SNR}_{\mathrm{in}}=\mathrm{R} / \mathrm{R}_{\mathrm{d}}=\mathrm{B} / \mathrm{B}_{\mathrm{d}}
$$




\section{$R_{s}$ is the transmission rate of spread spectrum code, $\mathbf{R}_{\mathbf{d}}$ is the transmission rate of information data, $\mathbf{B}_{\mathbf{s}}$ is the bandwidth of spread spectrum code, $\mathbf{B}_{\mathrm{d}}$ is the bandwidth of information data. \\ 3 Frequency Hopping Communication System}

Frequency hopping is to control the frequency synthesizer by using the pseudo code sequence to form the frequency synthesizer. The transmitted information code and the pseudo random sequence mode two (or the waveform multiplication) constitute the hopping instruction (i.e., the frequency hopping pattern), and by which the transmission frequency is randomly selected. The simplified block diagram of the frequency hopping communication system is shown in Figure 1. After modulation, the sender's information code sequence and the pseudo random sequence are controlled by different frequency hopping pattern. At the receiving end, the signal and noise received by the filter is sent to the mixer. The receiver's local oscillator signal is a frequency hopping signal, the same as the transmission end is the same, the frequency of the two frequency synthesizer is relatively, but the corresponding frequency has a frequency difference, just as the receiver of the intermediate frequency. As long as the sender and receiver of the pseudo-random code synchronization, it can make both the frequency hopping frequency synthesizer and the frequency synthesizer to generate the frequency synchronization, after mixing, you can get a constant frequency signal, and then the signal demodulation, you can recover the transmitted information. In the case of interference signal, the frequency of the frequency synthesizer is not related to the frequency of the frequency synthesizer. Therefore, it can not enter into the intermediate frequency channel, which can not interfere with the formation of the frequency hopping system.

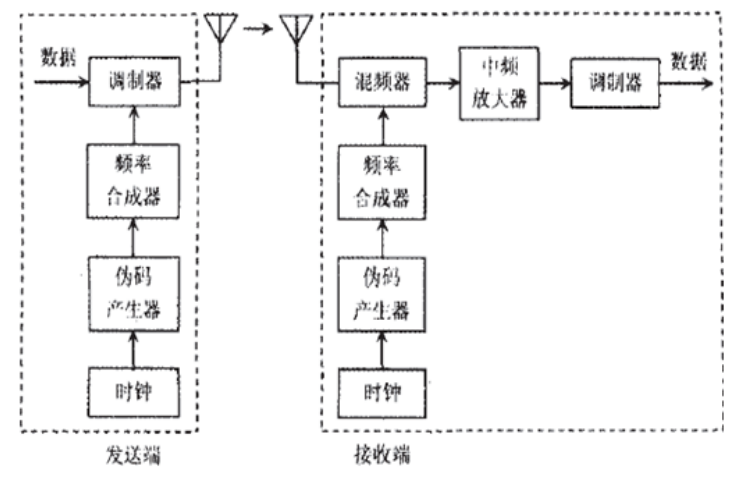

Figure 1 Frequency hopping communication system model

\section{Simulation Platform of MATLAB System}

MATLAB is a kind of mathematical application software, after many years of development, the development of the communication system, including the number of tools, become the most widely used in scientific research and engineering software. Simulink is a visual simulation tool in MATLAB, just click and drag the mouse operation to complete the simulation, it is simple and clear, and the user can immediately see the results of the simulation system. In addition, MATLab provides a program language based on matrix calculation, and it can also be shown by the function of writing the function of the combination of source program and graphics rendering.

Part in the MATLAB simulation procedures can be broadly divided into the majority of 6 , respectively, the main function, sending module, receiver module, AWNG channel, Walsh function and error counters.

In this paper, a simple simulation is made on the source code of a frequency hopping communication, and the time domain waveform and spectrum of the signal are analyzed.

Procedures are mainly composed of the following steps: first, through the rand function to generate a data signal carrier and a fixed frequency carrier signal signal, and then the two BPSK modulation, the modulation output is modulated signal BPSK. Frequency signal spread is generated by the stitch function. We choose 6 kinds of frequency (we consider 6 kinds of jump frequency to meet the requirements of the working process and the effect of frequency hopping). Finally, signal BPSK and modulated signal spread frequency hopping modulation, frequency hopping spread spectrum signal. 


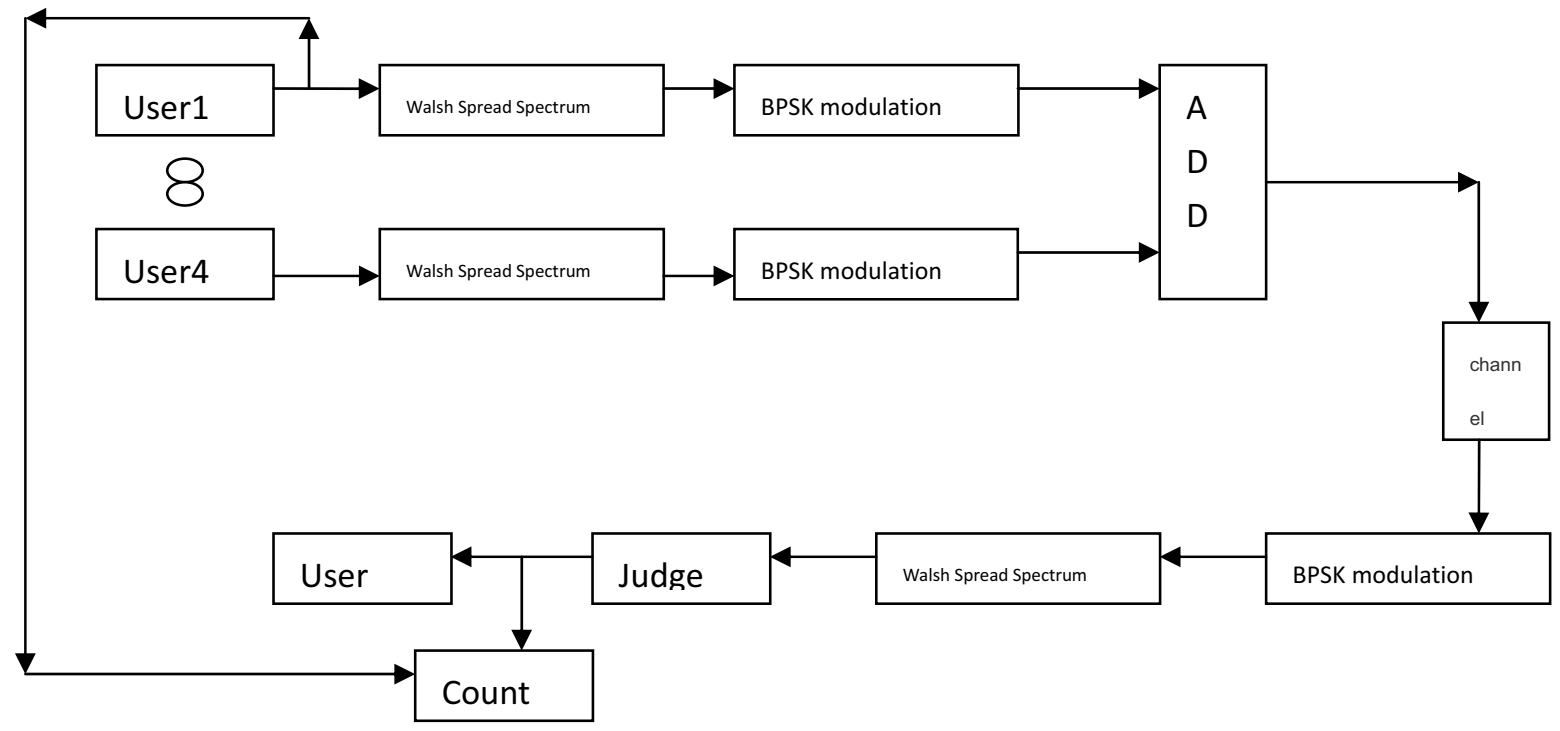

Figure 2 block diagram of simulation system

\section{Simulation Results}

Signal in frequency hopping spread spectrum before and after the waveform, the use of MATLAB simulation, as shown in figure 2: The original bit sequence is a randomly generated binary sequence as the data signal to be transmitted. After BPSK modulation, the BPSK modulated signal is obtained. Sread signal with 6 frequencies is a randomly generated FM signal.There are six kinds of frequency in the picture, frequency hopped spread spectrum signal is the output signal after frequency hopping modulation. The bandwidth is broadened, and the frequency components are increased and the frequency components are increased, so that the receiver is not aware of the original data.
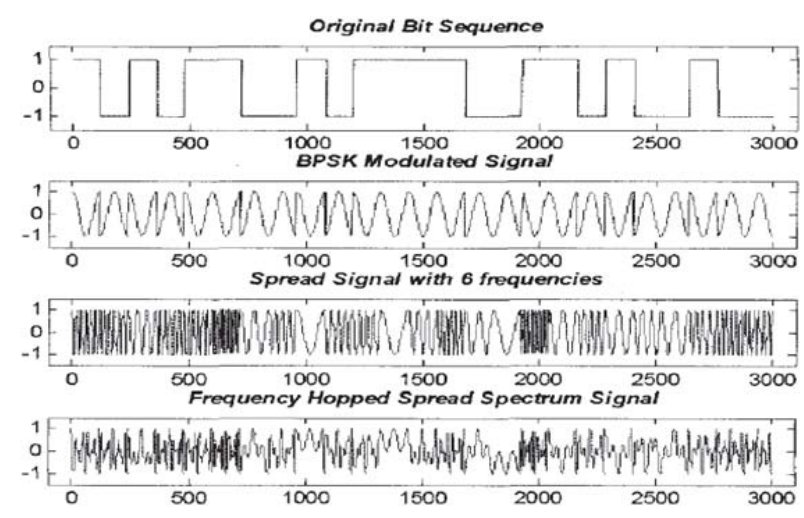

Figure 3 Frequency hopping communication signal

\section{Conclusions}

Frequency hopping communication is widely used in military and civilian fields because of its anti - jamming. At present, the popular Bluetooth technology and wireless LAN technology have the figure of frequency hopping technology. The modulation of transmitter in frequency hopping communication system through the MATLAB simulation analysis the principle of frequency hopping communication sender's working process and spread spectrum.

Building the frequency hopping system and simulation, and the error rate analysis. The theory basis of communication theory is based on the good advantages of frequency hopping communication:

1) considering the bandwidth and signal to noise ratio in order to find the optimal parameters, the maximum utilization of the anti-jamming performance is given;

2) the secrecy and non-distortion characteristics of frequency hopping communication.

3) frequency hopping communication uses a variety of patterns or clocks, so that the frequency resource sharing within a bandwidth to improve frequency utilization.

\section{References}

1. Junnian Wang,Jinfeng Wu. Based on Matlab / Simulink communication principle visual dynamic simulation [J]. Gansu Agricultural University 2003(6).

2. Ping Ping,Yicheng .Song Digital communications,Electronic Industry Press, 2008. 562

3. Zhenyu Li. Research on spread spectrum communications received coded modulation and related technologies [J] NORTHERN JIAOTONG UNIVERSITY newspaper, 1995,(04) 